\title{
La endogamia como causa de consanguinidad y su asociación con anomalías congénitas
}

\section{Endogamy as a cause of consanguinity and its association with congenital anomalies}

\author{
Daniela Torres-Hernández¹, Tatiana Fletcher-Toledo', \\ Roberth Alirio Ortiz-Martínez², María Amparo Acosta-Aragón ${ }^{3}$
}

\begin{abstract}
Resumen. El papel de la endogamia como causa de homocigosidad en la salud humana es un foco de interés en genética médica, debido a su relación con anomalías congénitas y patologías genéticas recesivas. Es un tema importante a pesar de que las tasas de uniones consanguíneas en ciertas sociedades han disminuido con el tiempo; sin embargo, en algunas comunidades se han mantenido estables o han aumentado. La consanguinidad es practicada hasta en el 10\% de la población mundial, y los motivos más comúnmente citados son socioculturales y socioeconómicos. Aunque se ha visto una disminución de esta práctica, probablemente por la migración urbana y el aumento de las tasas de educación, la consanguinidad continúa practicándose en todo el mundo. Los efectos más significativos sobre los resultados reproductivos se deben, principalmente, a condiciones hereditarias autosómicas recesivas, que también aumentan la frecuencia de algunos desórdenes médicos. El objetivo de esta revisión es dar a conocer la epidemiología y los factores predisponentes de la consanguinidad, así como presentar la evidencia actual de la asociación entre la consanguinidad originada en la endogamia y las anormalidades congénitas y patologías médicas como consecuencia de trastornos genéticos mendelianos. Se requiere un enfoque culturalmente apropiado para el asesoramiento genético en relación con la endogamia.
\end{abstract}

Palabras clave: endogamia, consanguinidad, efecto fundador, anomalías congénitas.

\footnotetext{
${ }^{1}$ Médicas, Residentes de Tercer año de Pediatría, Facultad de Ciencias de la Salud, Universidad del Cauca. Popayán, Colombia 2 Médico, Especialista en Ginecobstetricia, MSc en Epidemiología. Profesor, Departamento de Gineco-Obstetricia, Facultad de Ciencias de la Salud, Universidad del Cauca, Hospital Universitario San José. Popayán, Colombia.

${ }^{3}$ Médica, MSc en Biología-Genética Clínica, PhD en Genética de Poblaciones Humanas y Genética Forense. Profesora Titular, Departamento de Pediatría, Facultad de Ciencias de la Salud, Universidad del Cauca, Hospital Universitario San José. Popayán, Colombia. E-mail: maragon@unicauca.edu.co.

Conflicto de interés: los autores declaran que no tienen conflicto de interés.

Medicina \& Laboratorio 2021;25:409-418. https://doi.org/10.36384/01232576.354.

Recibido el 21 de agosto de 2020; aceptado el 2 de diciembre de 2020. Editora Médica Colombiana S.A., 2021ㅇ․
} 


\begin{abstract}
The role of consanguinity as a cause of homozygosity in human health is a focus of interest in medical genetics, due to its relationship with congenital anomalies and recessive genetic pathologies. This is an important issue since the rates of consanguineous unions in certain societies have decreased over time, but have remained stable or have increased in others. Consanguinity is practiced in up to $10 \%$ of the world population, and the most common reasons are sociocultural and socioeconomic factors. Although there has been a decrease in this practice, probably due to urban migration and an increase in education rates, consanguinity continues to be practiced throughout the world. The most significant effects on reproductive outcomes are mainly due to autosomal recessive hereditary conditions, that also increase the frequency of medical disorders. The aim of this review is to present the current evidence of the association between consanguinity originating from endogamy, with congenital abnormalities and medical disorders originated from mendelian genetic pathologies. A cultural appropriate approach is required for genetic counseling in relation to consanguineous endogamy.
\end{abstract}

Keywords: endogamy, consanguinity, founder effect, congenital anomalies.

\section{Introducción}

Se considera que entre 500 y 800 millones de personas en la población mundial actual practican matrimonios consanguíneos [1]. La consanguinidad puede ser el resultado del aislamiento o de las costumbres endogámicas en muchas comunidades. A su vez, el grado de aislamiento de las poblaciones está en función de dos parámetros: 1) la tasa de inmigración y, 2) el número de habitantes (en el caso de aislamiento geográfico) o el tamaño poblacional efectivo (en el caso de aislamiento reproductivo). Cuanto menor sea el tamaño de una población, más alta es la probabilidad de que un matrimonio sea consanguíneo, y es por esto que la genética médica tiene un foco de interés en el papel de la consanguinidad y la endogamia en la salud humana [2].

Se ha descrito que la endogamia y su consecuencia, la consanguinidad, están relacionadas con el aumento en el nivel de homocigotos para los trastornos autosómicos y las anomalías congénitas de origen genético o multifactorial. La prevalencia de la consanguinidad y el grado de endogamia varían de una población a otra dependiendo de la etnia, la religión, la cultura y la geografía. Los estudios epidemiológicos globales han revelado que las uniones consanguíneas se han asociado significativamente con una mayor susceptibilidad a diversas formas de enfermedades hereditarias [3].

El objetivo de este artículo es realizar una revisión sobre la endogamia como causa de consanguinidad y su relación con la presentación de anomalías congénitas y patologías médicas producto de trastornos mendelianos recesivos, con base en artículos publicados a nivel mundial y con algunos reportes de Colombia.

\section{Definiciones}

\section{Endogamia}

El diccionario la define como "el cruzamiento entre individuos de una raza, 
comunidad o población aislada genéticamente", y se caracteriza técnicamente como la condición homocigótica de genes en un determinado sitio (locus) cromosómico [4].

Conocer el grado de endogamia en los asentamientos humanos es importante para la salud pública, ya que se correlaciona con un alto riesgo de enfermedades genéticas de herencia recesiva $[5,6]$. Hay dos fuentes principales de endogamia; la primera son los aislados genéticos, que se observan en las poblaciones pequeñas sometidas a una fuerte deriva génica, y por lo tanto, a homogeneización genética concurrente dentro de las cuales hay pocas opciones de apareamiento [7]. La segunda, se debe a la característica cultural de ciertos grupos étnicos, que consiste en uniones conyugales preferenciales entre miembros de una misma familia, lo que genera grupos genéticamente diferenciados dentro de las comunidades [8,9]. En ambos casos, el número de loci genéticos en homocigosis se ve incrementado.

\section{Consanguinidad}

El término "consanguinidad" es derivado de la palabra en latín consanguineus, que significa de la misma sangre; "con" (juntos) y "sanguis" (sangre) [10].
El término "consanguinidad" no debe confundirse con los términos "endogamia" o "incesto". En la práctica clínica se usa a menudo para describir uniones entre individuos que comparten al menos un ancestro en común, y puede ser consecuencia de la endogamia. Como las personas que viven en la misma comunidad suelen compartir al menos un ancestro común en su genealogía, el término "consanguinidad" en genética médica se restringe a las uniones entre parientes de quinto grado, como primos segundos, o más cerca $[11,12]$ (tabla 1).

\section{Efecto fundador}

El efecto fundador es un fenómeno que se refiere a la migración de un pequeño grupo de personas de una población mayor para establecerse en otro entorno, llevando consigo una parte de la información genética que existía en la población original. Debido a que todos pertenecen a un subconjunto, reducen la cantidad de variabilidad genética que existe dentro de la nueva población. Como resultado de ello, ciertos fenotipos o determinados genes, a veces con consecuencias patológicas (tener o no una enfermedad en particular), pueden verse resaltados o atenuados en esta población $[10,13]$.

Tabla 1. Grados de parentesco y genes en común

\begin{tabular}{llc}
\hline Grado de parentesco & \multicolumn{1}{c}{ Tipo } & Genes en común \\
\hline Primero & Padres-hijos, hermanos, gemelos dicigóticos & $50 \%$ \\
Segundo & $\begin{array}{l}\text { Hermanos-medios, tíos-sobrinos, abuelos- } \\
\text { nietos }\end{array}$ & $25 \%$ \\
Tercero & Primos hermanos, bisabuelos-bisnietos & $12,5 \%$ \\
Cuarto & Primos hermanos un grado aparte, primos & $6,25 \%$ \\
Quinto & hermanos medios & $3,125 \%$ \\
\hline
\end{tabular}




\section{Factores predisponentes de consanguinidad}

Las razones dadas para la preferencia de los matrimonios consanguíneos son principalmente sociales. En las comunidades con altos índices de consanguinidad, los estudios sociológicos indican que los matrimonios consanguíneos podrían ser por "estabilidad" debido a una mayor compatibilidad entre marido y mujer, ya que comparten las mismas relaciones sociales, así como la compatibilidad con otros miembros de la familia [14]. También indican mejor relación con su familia política, ya que podría servir de apoyo en tiempo de necesidad. Además, existe la creencia general de que casarse dentro de la familia reduce las posibilidades de incertidumbres ocultas en cuestiones de salud y financieras, por lo que también incluye motivos económicos $[10,14]$.

Se cree que la consanguinidad fortalece los lazos familiares y hace cumplir la solidaridad familiar, proporcionando excelentes oportunidades para la transmisión de los valores culturales y continuidad cultural; sin embargo, la frecuencia de "matrimonios arreglados" puede estar disminuyendo en los últimos años, debido al aumento del número de mujeres que alcanzan la educación universitaria, lo cual les da una elección más amplia de la pareja matrimonial [15].

La consanguinidad tiene alguna base religiosa, y este es un factor importante que influye en la prevalencia en algunas comunidades. Ciertas religiones tienen mayor predisposición a la consanguinidad. Por ejemplo, en el judaísmo y el budismo se sancionan las uniones consanguíneas. Las actitudes hacia la consanguinidad dentro del islam son ambiguas, pero se piensa que, en general, el islam favorece las uniones consanguíneas [16].

\section{Epidemiología}

Aproximadamente el $10 \%$ de la población mundial es de origen consanguíneo $[1,9,17]$. Dependiendo de los antecedentes culturales, legales y religiosos, la prevalencia de la consanguinidad varía en las diferentes regiones, con tasas entre el $20 \%$ y el $50 \%$ en el Medio Oriente y norte de África, entre el $1 \%$ y el $5 \%$ en el sur de Europa, América del Sur y Japón, y menos del 1\% en Europa Occidental, América del Norte y Oceanía [18] (figura 1). También se han descrito altos niveles de consanguinidad en algunas comunidades cerradas como los amish de los Estados Unidos y los samaritanos de Jordania e Israel [19].

El primer estudio que informó sobre un paciente heterocigoto compuesto, cuyos padres estaban relacionados y vivían en un aislado genético, se originó en los Países Bajos [20]. La historia demográfica de estos países está caracterizada por la inmigración principalmente de marroquíes y turcos, y el porcentaje de mujeres con parejas consanguíneas es de $24 \%$ en turcos, $22 \%$ en marroquíes y $0,1 \%$ en holandeses nativos. No se sabe con precisión cuántos niños con enfermedades autosómicas recesivas nacen cada año de padres consanguíneos en los Países Bajos, pero se puede hacer una suposición tomando el número anual de niños nacidos de madres turcas o marroquíes (15.000 según la oficina de estadística holandesa), y asumiendo que existe una frecuencia del $25 \%$ de matrimonios consanguíneos, con un riesgo de $1 \%$ de enfermedades autosómicas recesivas en este grupo, da como resultado unos 40 niños por año [21]. 


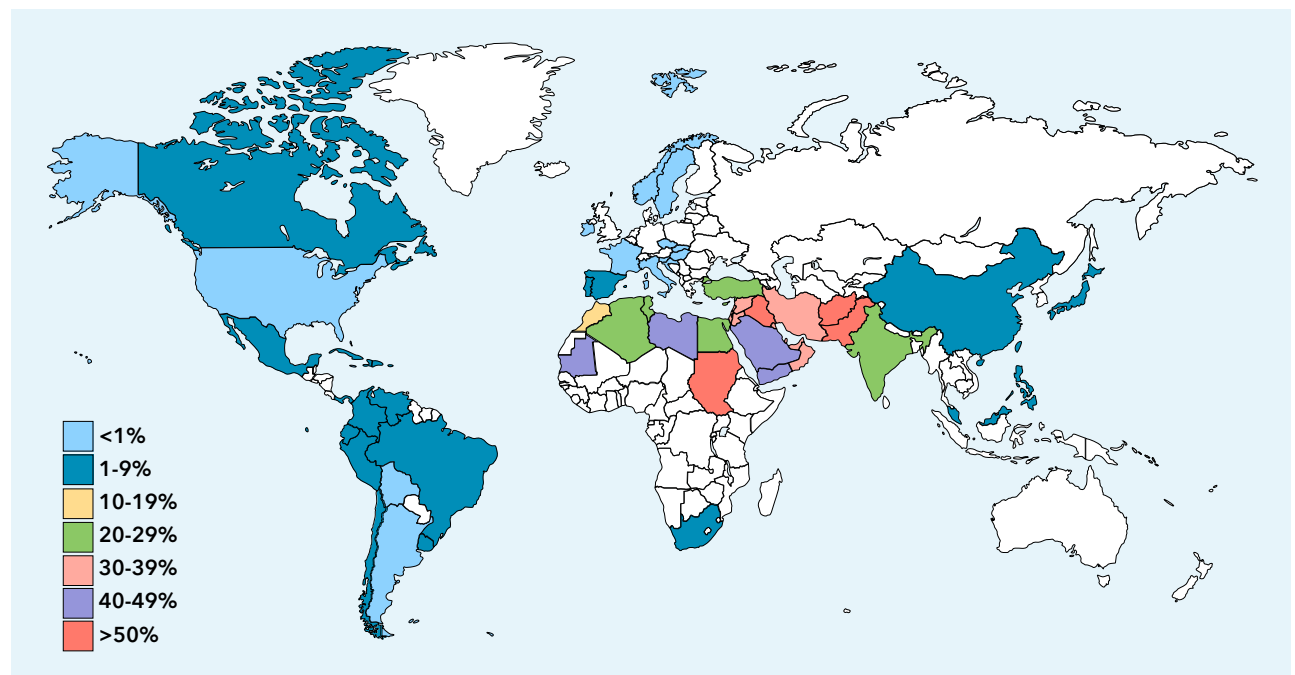

Figura 1. Tasas de matrimonios consanguíneos a nivel mundial, donde se incluyen los matrimonios entre primos segundos o parientes más cercanos [1].

Un estudio realizó una comparación de la consanguinidad a lo largo de 49 años (1918-1966) en diversas zonas del país Vasco [22]. Tres poblaciones se destacaron por su frecuencia de matrimonios consanguíneos: Alda $(23,53 \%)$, Contrasta (20\%) y Arlucea $(10,07 \%)$. Este trabajo concluye que la base para la evolución diferenciada de ciertos hechos demográficos, como la mortalidad infantil o la densidad variable de ciertas enfermedades, en estas poblaciones, eran los genes homocigóticos heredados.

Se evaluó también la magnitud del aislamiento genético y las costumbres de endogamia en 57 comunidades de tres municipios rurales de Honduras, utilizando técnicas de isonimia (apellidos en común). Encontraron que las comunidades estudiadas presentaban alta consanguinidad debido al aislamiento, la segregación étnica y las costumbres endogámicas [2].

En América del Sur, un estudio demostró que, en promedio, solo el $1 \%$ de las uniones conyugales eran consanguíneas, siendo las tasas más altas las observadas en Venezuela $(1,84 \%)$ y Brasil $(1,60 \%)$, en tanto que las más bajas se observaron en Argentina (0,29\%) y Bolivia (0,31\%). En Colombia, en particular, la tasa fue del 1,31\% [23].

\section{Anomalías congénitas y patologías asociadas a consanguinidad}

Son numerosos los estudios que han evaluado la relación entre la consanguinidad y la incidencia de defectos del nacimiento, y la mayoría de los estudios han determinado una asociación positiva significativa y consistente [8]. Los hijos de parejas consanguíneas tienen un mayor riesgo de defectos congénitos, ya que el mismo gen mutante puede heredarse de ambos padres. Cuanto más estrecha sea la relación biológica entre los padres, mayor será la probabilidad de que su progenie herede el mismo gen recesivo desfavorable. 
La unión consanguínea es uno de los eventos relacionados con la cultura en todo el mundo, particularmente en los países de bajos ingresos [18], e incluso ha sido reportada como la causa más importante de mortalidad asociada a las anomalías congénitas [3]. Varios estudios indican que la prevalencia de anomalías congénitas mayores en países desarrollados es el $2 \%$ al $3 \%$ y puede llegar hasta un $7 \%$ en países en vía de desarrollo [24]. Las parejas consanguíneas son más propensas a experimentar la muerte de una descendencia, que aquellas que no están relacionadas [3].

Las anomalías que se relacionan con mayor frecuencia a la consanguinidad incluyen las cardiovasculares, los defectos de cara y cuello, afectación del sistema músculo-esquelético, aparato digestivo, aparato genitourinario, sistema nervioso central, defectos del tubo neural, pérdida de audición congénita y defectos visuales [25-28]. Sin embargo, también se ha encontrado asociación con otras alteraciones como inmunodeficiencia, ß-talasemia, hipertensión arterial en los niños y bajo peso al nacer, entre otras [8]. Bromiker y colaboradores determinaron que en Jerusalén la incidencia de anomalías congénitas mayores en los niños de uniones de primos hermanos, era tres veces mayor $(8,7 \%)$ que en los recién nacidos de padres que no estaban relacionados (2,6\%) [29]. En cuanto a las alteraciones cardiacas, en particular, un estudio realizado en Egipto encontró que la comunicación interventricular (OR=2,70; IC95\%:2,07-3,50) y la comunicación interauricular $(\mathrm{OR}=2,87$; IC95\%:1,85-4,47) se encontraban fuertemente influenciadas por la consanguinidad [30]. Nabulsi y colaboradores en Líbano, informaron de una asociación significativa entre la consanguinidad y las enfermedades cardiacas congénitas, incluyendo anomalías valvulares aórticas, comunicación interauricular e interventricular, tetralogía de Fallot y estenosis pulmonar, en matrimonios entre primos de primer grado [31].

Un estudio en Arabia Saudita, que fue publicado en 2015, encontró una consanguinidad del $49,6 \%$ en las mujeres incluidas en el estudio $(n=2.441)$, la cual demostró ser un factor de riesgo independiente para el desarrollo de los síndromes genéticos observados en los recién nacidos [32]. De manera similar, en otro estudio en la misma región, se reportó una prevalencia de anomalías congénitas de 412/10.000 nacimientos [33]. Las más frecuentes fueron las cardiopatías congénitas, las malformaciones renales y los defectos del tubo neural, encontrándose una asociación significativa entre estas anomalías y la consanguini$\operatorname{dad}(\mathrm{OR}=1,5$; IC95\%:1,28-1,81).

El paladar hendido y labio leporino son las malformaciones congénitas más comunes de la cabeza y cuello en Irán. En un estudio realizado en 2017, se encontró que el matrimonio consanguíneo se asocia fuertemente (OR=3,2; IC95\%:1,9$5,3)$ con una mayor incidencia de esta anomalía [34], en tanto que en Palestina se encontró que en los pacientes con paladar hendido la tasa de consanguinidad era del 67\% [35].

En Irak, Ameen y colaboradores incluyeron 130 mujeres que tuvieron recién nacidos con al menos una anomalía congénita. Las anomalías más frecuentes fueron del sistema nervioso central $(37,7 \%)$, seguido de los sistemas musculoesquelético $(23,1 \%)$ y gastrointestinal (20,8\%). Hubo una asociación significativa entre las anomalías congénitas halladas y la consanguinidad de los padres (OR=6,26; IC95\%:2,42-16,19) [36]. 


\section{Anomalías asociadas a consanguinidad en Colombia}

Antes del surgimiento de las pruebas de ADN, los estudios genéticos utilizaban los apellidos como marcadores de la estructura de la población en términos de parentescos. La relación entre apellidos repetidos y patologías coincide con los resultados hallados en el 2006 en Colombia [37], donde se muestra que los apellidos repetidos y uniones consanguíneas están asociados a la ocurrencia de diversas patologías como fibrosis quística, albinismo y trastorno afectivo bipolar.

Se conocen 36 casos en Colombia con mucopolisacaridosis tipo $\mathrm{VI}$, de los cuales 10 están en un grupo indígena, lo que constituye un aislado genético por vivir dentro de resguardos establecidos desde la época colonial [38]. En la investigación realizada por Acosta y colaboradores se estudiaron dos de estos pacientes con la forma severa de mucopolisacaridosis, por genética molecular, provenientes del suroeste de Colombia, en el departamento del Cauca. En los dos casos se encontró una transversión patogénica de un solo nucleótido (p.C447F), que produce una mutación de cambio de sentido en el exón 8 del gen ARSB. Los dos pacientes, casos índices en sus respectivos núcleos familiares, tuvieron la misma mutación, lo cual sugirió la posibilidad de un alelo ancestral común, probablemente debido a la endogamia relativa y al aislamiento geográfico histórico de estas regiones [38].

En las regiones de Yarumal, Belmira y Canoas, en el departamento de Antioquia, se estudiaron tres pacientes de diferentes familias con demencia tipo Alzheimer y antecedentes de demencia familiar. Al realizarse las genealogías, se encontraron un total de 86 casos. Las tres familias compartieron en alta proporción cuatro apellidos, lo cual hace suponer que posiblemente se trate de una sola familia. La frecuencia del gen PPA mutado fue de aproximadamente 0,018 para la población estudiada; es decir, que aproximadamente el $2 \%$ de la población era portadora del gen para la demencia tipo Alzheimer. Este estudio se consideró como la serie más grande reportada de demencia tipo Alzheimer con agregación familiar hasta la fecha [39].

En el estudio realizado por Ospina y colaboradores, se identificaron 28 familias, todas originarias del oriente del departamento de Antioquia, con trastorno afectivo bipolar tipo I, donde se encontró ligamiento significativo en el $100 \%$ de los casos [40]. En otro estudio, realizado en una gran familia en Peque, Antioquia, se encontraron un total de 163 individuos afectados por la enfermedad de Parkinson juvenil. La mutación reportada (G736A), localizada en el exón 6 del gen PARK2, tiene una alta frecuencia en esta región, debido a un posible efecto fundador, al crecimiento interno y a la existencia de cruces endogámicos [41].

Finalmente, en el departamento del Cauca, en 2012, se realizó un estudio observacional de casos y controles con casos incidentales de anomalías congénitas. Se encontró que las más frecuentes fueron de tipo multisistémico $(18,4 \%)$, del sistema gastrointestinal (14,3\%), cardiovascular (11,2\%), osteomuscular (11,2\%) y craneofacial (11,2\%). En el análisis bivariado se encontró asociación significativa con la consanguinidad o procedencia de una misma vereda $(\mathrm{OR}=1,4 ; \mathrm{IC} 95 \%: 1,0$ 1,9) [42].

\section{Conclusiones}

La consanguinidad continúa practicándose en todo el mundo, y en algunos países las tasas siguen aumentando. 
Los estudios realizados sobre consanguinidad y endogamia, la mayoría retrospectivos de cohorte y de casos y controles, han permitido establecer una asociación significativa entre la consanguinidad y el desarrollo de anomalías congénitas. La consanguinidad también ha sido relacionada con un mayor riesgo de producir descendientes que son homocigotos para un gen mutado recesivo, y a una mayor susceptibilidad a enfermedades multifactoriales o poligénicas, enfermedades recesivas, abortos espontáneos y mortinatos. Se requiere un enfoque culturalmente apropiado para el asesoramiento genético en relación con los matrimonios consanguíneos y la endogamia.

\section{Referencias}

1. Oniya O, Neves K, Ahmed B, Konje JC. A review of the reproductive consequences of consanguinity. Eur J Obstet Gynecol Reprod Biol 2019;232:87-96. https://doi.org/10.1016/j. ejogrb.2018.10.042

2. Herrera-Paz EF. Aislamientos genéticos y costumbres endogámicas en tres municipios rurales de Honduras. Rev Med Inst Mex Seguro Soc 2016;54:504-513.

3. Fareed M, Afzal M. Genetics of consanguinity and inbreeding in health and disease. Ann Hum Biol 2017;44:99-107. https://doi.org/10. 1080/03014460.2016.1265148.

4. Márquez-Sánchez F. ¿Para qué sirve la endogamia? Comunicaciones libres. Ciencia 2007; 58:72-78.

5. Charlesworth D, Willis JH. The genetics of inbreeding depression. Nat Rev Genet 2009;10:783-796. https://doi.org/10.1038/ nrg2664.

6. Bittles AH, Neel JV. The costs of human inbreeding and their implications for variations at the DNA level. Nat Genet 1994;8:117-121. https://doi.org/10.1038/ng1094-117.

7. Sherwin JC, Hewitt AW, Ruddle JB, Mackey DA. Genetic isolates in ophthalmic diseases.
Ophthalmic Genet 2008;29:149-161. https:// doi.org/10.1080/13816810802334341.

8. Bhinder MA, Sadia H, Mahmood N, Qasim M, Hussain Z, Rashid MM, et al. Consanguinity: A blessing or menace at population level? Ann Hum Genet 2019;83:214-219. https://doi. org/10.1111/ahg.12308.

9. Hamamy H. Consanguineous marriages: Preconception consultation in primary health care settings. J Community Genet 2012;3:185-192. https://doi.org/10.1007/s12687-011-0072-y.

10. López-Valdez JA, Báez-Reyes MR. Asesoramiento genético en parejas consanguíneas. Rev Sanid Milit Mex 2009;63:308-315.

11. Bennett RL, Motulsky AG, Bittles A, Hudgins L, Uhrich S, Doyle DL, et al. Genetic counseling and screening of consanguineous couples and their offspring: Recommendations of the national society of genetic counselors. J Genet Couns 2002;11:97-119. https://doi. org/10.1023/A:1014593404915.

12. Saggar AK, Bittles AH. Consanguinity and child health. Paediatr Child Health 2008;18:244-249. https://doi.org/10.1016/j. paed.2008.02.008.

13. Giddings LV, Kaneshiro KY. Genetics, speciation and the founder principle. Evolution 1984;38:115. https://doi. org/10.1111/j.1558-5646.1984.tb00264.x.

14. Ben-Omran T, Al Ghanim $K$, Yavarna T, EI Akoum M, Samara M, Chandra $P$, et al. Effects of consanguinity in a cohort of subjects with certain genetic disorders in Qatar. Mol Genet Genomic Med 2020;8:e1051. https:// doi.org/10.1002/mgg3.1051.

15. Pyeritz R, Korf B, Grody W. Emery and Rimoin's Principles and Practice of Medical Genetics and Genomics. Clinical principles and applications. 7th ed. Ámsterdam, Países Bajos: Elsevier; 2018. p. 412. ISBN: 9780128125366.

16. Tadmouri GO, Nair P, Obeid T, Al Ali MT, Al Khaja N, Hamamy HA. Consanguinity and reproductive health among Arabs. Reprod Health 2009;6:17. https://doi. org/10.1186/1742-4755-6-17.

17. Schulpen TWJ, van Wieringen JCM, van Brummen PJ, van Riel JM, Beemer FA, Westers $\mathbf{P}$, et al. Infant mortality, ethnicity, and genetically determined disorders in The Nether- 
lands. Eur J Public Health 2005;16:290-293. https://doi.org/10.1093/eurpub/cki201.

18. Soheilirad Z. What should be the focus of counseling in parental consanguinity: genetic disorders or underlying beliefs. Egypt J Med Hum Genet 2020;21:8. https://doi.org/10.1186/ s43042-020-0049-7.

19. Jaber L, Halpern GJ, Shohat M. The impact of consanguinity worldwide. Community Genet 1998;1:12-17. https://doi. org/10.1159/000016130.

20. Ten-Kate LP, Scheffer H, Cornel MC, van Lookeren-Campagne JG. Consanguinity sans reproche. Hum Genet 1991;86:295-296. https://doi.org/10.1007/bf00202413.

21. Ten-Kate LP, Teeuw ME, Henneman L, Cornel MC. Consanguinity and endogamy in the netherlands: Demographic and medical genetic aspects. Hum Hered 2014;77:161-166. https://doi.org/10.1159/000360761.

22. Zudaire-Huarte C. Coeficiente de consanguinidad en zonas de Álava, Guipúzcoa y Vizcaya. Munibe Antropol-Arkeol 1981;33:245-254.

23. Liascovich R, Rittler M, Castilla EE. Consanguinity in South America: Demographic aspects. Hum Hered 2001;51:27-34. https://doi. org/10.1159/000022956.

24. Ávila-Mellizo GA. Defectos Congénitos. Colombia 2018. Bogotá, Colombia: Instituto Nacional de Salud; 2018. p. 39. Acceso 28 de junio de 2020. Disponible en https://www.ins. gov.co/buscador-eventos/Informesdeevento/ DEFECTOS\%20CONG\%C3\%89NITOS_2018. pdf.

25. Bowirrat A, Armaly Z. Consanguineous marriages in the Middle East: Nature versus nurture. Open Complement Med J 2014;5:1-11. https://doi.org/10.2174/187639 1X01305010001.

26. Stoltenberg $C$, Magnus $P$, Lie RT, Daltveit AK, Irgens LM. Birth defects and parental consanguinity in Norway. Am J Epidemiol 1997;145:439-448. https://doi.org/10.1093/ oxfordjournals.aje.a009126.

27. Ramegowda S, Ramachandra NB. Parental consanguinity increases congenital heart diseases in South India. Ann Hum Biol 2006;33:519-528. https://doi. org/10.1080/03014460600909349.
28. Shieh JTC, Bittles AH, Hudgins L. Consanguinity and the risk of congenital heart disease. Am J Med Genet A 2012;158A:1236-1241. https://doi.org/10.1002/ajmg.a.35272.

29. Bromiker $R$, Glam-Baruch $M$, Gofin $R$, Hammerman C, Amitai Y. Association of parental consanguinity with congenital malformations among Arab newborns in Jerusalem. Clin Genet 2004;66:63-66. https://doi.org/10.1111/ j.0009-9163.2004.00264.x.

30. Bassili A, Mokhtar SA, Dabous NI, Zaher SR, Mokhtar MM, Zaki A. Risk factors for congenital heart diseases in Alexandria, Egypt. Eur J Epidemiol 2000;16:805-814. https://doi. org/10.1023/A:1007601919164.

31. Nabulsi MM, Tamim H, Sabbagh $M$, Obeid MY, Yunis KA, Bitar FF. Parental consanguinity and congenital heart malformations in a developing country. Am J Med Genet 2003;116A:342347. https://doi.org/10.1002/ajmg.a.10020.

32. Majeed-Saidan MA, Ammari AN, AlHashem AM, Al Rakaf MS, Shoukri MM, Garne E, et al. Effect of consanguinity on birth defects in Saudi women: results from a nested case-control study. Birth Defects Res A Clin Mol Teratol 2015;103:100-104. https://doi.org/10.1002/ bdra.23331.

33. Kurdi AM, Majeed-Saidan MA, Al Rakaf MS, AlHashem AM, Botto LD, Baaqeel HS, et al. Congenital anomalies and associated risk factors in a Saudi population: a cohort study from pregnancy to age 2 years. BMJ Open 2019;9:e026351. https://doi.org/10.1136/bmjopen-2018-026351.

34. Jamilian A, Sarkarat F, Jafari M, Neshandar M, Amini E, Khosravi S, et al. Family history and risk factors for cleft lip and palate patients and their associated anomalies. Stomatologija 2017;19:78-83.

35. Saeed OB, Moore MG, Zawahrah H, Tayem M, Kavoosi T, van Aalst JA. The Influence of consanguinity on familial clefting among Palestinians. Cleft Palate Craniofac J 2019;56:1072-1079. https://doi. org/10.1177/1055665619838381.

36. Ameen SK, Alalaf SK, Shabila NP. Pattern of congenital anomalies at birth and their correlations with maternal characteristics in the maternity teaching hospital, Erbil city, Iraq. BMC 
Pregnancy Childbirth 2018;18:501. https:// doi.org/10.1186/s12884-018-2141-2.

37. Bedoya G, García J, Montoya P, Rojas W, Amézquita ME, Soto I, et al. Análisis de isonimia entre poblaciones del noroeste de Colombia. Biomédica 2006;26:538-545. https://doi. org/10.7705/biomedica.v26i4.323.

38. Acosta-Aragón MA, Lago-Lestón RM, Barros-Angueira F, Carracedo-Alvaez AM. Same mutation in two patients of southwestern colombia with mucopolysaccharidosis type $\mathrm{VI}$ or Maroteaux-Lamy syndrome: is there an endogamic effect? Genet Cell Biol 2019;2:82-86.

39. Lopera F, Arcos M, Madrigal L, Kosik K, Cornejo W, Ossa J. Demencia tipo Alzheimer con agregación familiar en Antioquia, Colombia. Acta Neurol Colomb 1994;10:173-186.
40. Ospina-Duque J, Ochoa I, García J, López C, Calle J, Carvajal L, et al. Loci genéticos asociados al trastorno bipolar. Estudios en población colombiana. Rev Colomb Psiquiatr 2001;30:239-248.

41. Arias $P$ WH, Rojas $W$, Moreno $S$, Lopera F, Ruís-Linares A, Bedoya G. Origen de la mutación G736A del gen parkin en la población de Peque (noroccidente de Antioquia). Rev Colomb Antropol 2012;48:81-95.

42. Bravo-Gallego LY, Teherán-Bravo JM, Pantoja-Chamorro FI, Díaz-Castro R, AcostaAragón MA. Factores asociados a anomalías congénitas en neonatos del Cauca. Pediatría 2012;45:47-58. http-s://doi.org/https://doi. org/10.1016/S0120-4912(15)30005-7. 